\title{
Analysis of Potential Co-Benefits for Bicyclist Crash Imminent Braking Systems*
}

\author{
David H. Good, Kerry Krutilla \\ Indiana University \\ School of Public and Environmental Affairs \\ Bloomington, Indiana, USA \\ good@indiana.edu
}

\begin{abstract}
In the US, the number of traffic fatalities has had a long term downward trend as a result of advances in the crash worthiness of vehicles. However, these improvements in crash worthiness do little to protect other vulnerable road users such as pedestrians or bicyclists. Several manufacturers have developed a new generation of crash avoidance systems that attempt to recognize and mitigate imminent crashes with non-motorists. While the focus of these systems has been on pedestrians where they can make meaningful contributions to improved safety [1], recent designs of these systems have recognized mitigating bicyclist crashes as a potential co-benefit. This paper evaluates the performance of one system that is currently available for consumer purchase. Because the vehicle manufacturer does not claim effectiveness for their system under all crash geometries, we focus our attention on the crash scenario that has the highest social cost in the US: the cyclist and vehicle on parallel paths being struck from behind. Our analysis of co benefits examines the ability to reduce three measures: number of crashes, fatalities, and a comprehensive measure for social cost that incorporates morbidity and mortality. Test track simulations under realistic circumstances with a realistic surrogate bicyclist target are conducted. Empirical models are developed for system performance and potential benefits for injury and fatality reduction. These models identify three key variables in the analysis: vehicle speed, cyclist speed and cyclist age as key determinants of potential co-benefits. We find that the evaluated system offers only limited benefits for any but the oldest bicycle riders for our tested scenario.
\end{abstract}

Keywords-Bicyclist Crash Imminent Braking, Benefit Analysis, Pre-Crash System Performance Analysis

\section{INTRODUCTION}

In the US, the number of traffic fatalities has had a long term downward trend as a result of advances in the crash worthiness of vehicles. These improvements in crash worthiness do little to help protect non-motorists. Over the last few years, this has been reflected in the number of pedestrian and bicyclist fatalities increasing in both absolute and relative terms (up over $15 \%$ since 2010 for pedestrians and nearly $20 \%$ for bicyclists). In 2010 there were 618 bicyclists killed and an estimated 52,000 bicyclist injuries. This increased to 749 bicyclist fatalities in 2013 and 818 in 2015 with an estimated 45,000 injuries $[2,3,4]$. These trends are summarized in Figure 1.

\author{
Stanley Chien, Lingxi Li, and Yaobin Chen \\ Indiana University - Purdue University Indianapolis \\ Dept. of Computer and Electrical Engineering \\ Indianapolis, Indiana, USA
}



Figure 1. US traffic fatality rate and the percentage of bicyclist fatalities over time

While increasing and becoming more lethal, US, bicycle crashes are a relatively small part of the road safety with $2.3 \%$ of roadway fatalities. In most of Europe and China bicyclist fatalities compared to total fatalities is approximately $10 \%$. In Japan, bicyclists make up $14 \%$ of traffic fatalities. In Netherlands, they comprise $32 \%$ of roadway fatalities [5].

Around the world, the typical cyclist varies widely. In the US, there is a tendency for bicyclist crashes to be viewed as something that affects children. Figure 2, shows that the demographics of those killed in bicycle crashes has changed dramatically over time. Over the last forty years,, the average cyclist fatality has changed from mid-teens to over 45 as more



Figure 2. Demographic composition of bicyclist fatalities (FARS 1975-2015) 
older Americans turn to cycling as a form of fitness and recreation rather than mobility.

The objective for safer vehicles has also changed from crashworthiness to crash avoidance. The first generation of crash imminent braking systems (CIB) focused on preventing rear end collisions. Detecting these imminent crashes is relatively simple. In comparison, the newer generations of CIB systems have been developed to recognize and mitigate nonmotorist collisions where victims are harder to detect and behaviors much more complex.

In a previous paper we evaluated the benefits associated with CIB systems designed to mitigate pedestrian crashes [1]. We follow a similar approach here. Pedestrian crash geometries are more complex since they have a wider range of behaviors than are exhibited in rear end collisions and these behaviors can affect system performance quite a bit. Bicyclist crashe geometries are still more complex since bicycles bring significant speed to the crash and have a wider variety of maneuvers (they appear from the street or sidewalk, they may execute turns, etc.) Cyclists can legally occupy the travel lane and in some cases the sidewalk. As a result, many more scenarios need to be tested to fully evaluate system capabilities.

Evaluation of the full range of scenarios is beyond the scope (and budget) of our project and instead we focus on the potential to mitigate social loss in only the most important scenarios. The test track simulation approach we take is likely to be the only practical mechanism for evaluation since bicycle crashes are rare and statistical power from field operational trials is extremely low.

The rest of the paper is organized as follows. In Section II we develop a classification scheme for bicycle crash scenarios based on publicly available data. These are validated with GES from GES/FARS for 2015. In Section III, we briefly describe our models for determining the consequences of particular crashes. We also estimate a performance model based on 100 test track simulations using our surrogate target. In Section IV we develop models to be used for evaluating the effectiveness of the tested CIB system. In Section V we discuss the results and develop a set of conclusions and some steps for future work. Our analysis is limited by key missing variables. As a result, we can only talk about the performance of the system uncer best case assumptions and interpret our findings as the "potential benefits" of this type of CIB system. We find that under the best of circumstances that the benefits are rather limited, and are most likely to accrue only to the oldest cyclists because of their frailty.

\section{Testable Crash Scenarios Based on Publicly AVAILABLE DATA}

\section{A. Defining CIB Relevant Crashes}

Our analysis starts with GES (General Estimates System) and FARS (Fatal Accident Reporting System) for 2014 and 2015. We restrict our use to this data because they are more closely harmonized than in previous years, they provide a better evaluation of fatalities and are similar to the results produced using other approaches and provide sufficient detail about the bicycle crash geometry. For these two years there were on average an annual number of 54,000 bicyclist involved crashes (from GES) and include involvement by 56,000 cyclists. The average annual number of fatalities is 766 . Our analysis also includes a measure of social cost that comprises the tangible costs associated with a crash (congestion, property damage, lost wages, loss of functional capacity as well as the intangible costs (loss of life or diminished enjoyment of life) that are based on quality adjusted life year analysis. DOT regulatory guidance offers 9.1 million dollars as the complete value for a lost life [6]. This value of life incorporates what people would be willing to pay or forego to achieve a small reduction in the probability of life lost. It is not an accounting measure of lost wages or insurance settlements. The document also provides guidance about how to value injuries based on Abbreviated Injury Scale values (AIS). Fatalities comprise $62 \%$ of the $\$ 10$ billion annual social cost of bicycle crashes.

Our analysis narrows down the number of CIB relevant crashes that can be reasonably evaluated by eliminating some crashes 1) involving a single vehicle and a single non-motorist who is a cyclist; 2) only light vehicles in transport (no parked cars, heavy trucks, busses, motorcycles, special or emergency vehicles); 3 ) vehicle in forward motion that has not lost control or recovering from another evasive maneuver; 4) crashes where the bicyclist strikes the motor vehicle from behind. The remaining crashes comprise about $70 \%$ of the crashes, fatalities and social cost.

\section{B. Classification of Bicyclist Crashes}

We base the testable scenarios for bicyclist CIB systems on two different analyses: harmonized GES/FARS data for 20102011, a period where there was enhanced data collection for both pedestrians and more extensively for bicyclists; 2) some state level data for Michigan and Indiana where we have access to the police crash reports (PAR) and can reevaluate the coding of crashes as a form of quality control.

It is important to keep in mind that the purpose of the PAR is not as a scientific instrument, but to determine liability and potential criminal fault surrounding the crash. Neither pedestrian nor cyclist crashes are systematically investigated as a part of the Federal data gathering process. These PAR are the sole source of information for both GES and FARS. From out analysis of data with both actual PAR information and the way that they were coded, we find that many important features are often not described in the PAR crash narrative and key features may be left of out the report since they are unrelated to culpability.

Our approach is to this potentially missing data is to develop a three tier classification scheme for bicycle crashes. The first tier is gross geometry, tier two includes vehicle maneuver and the third tier involves cyclists maneuvers. We find that it is almost always possible to identify tier 1 from the PAR, usually possible to identify tier 2 classifications and sometimes tier 3 is a hunch based on subtleties of the scene diagram. While these are sometimes little more than a hunch. These are summarized in Figure 3.

As a way to shorthand sometimes complex scenarios we use either CP, PP or UP to classify tier 1 (crossing path, parallel path or unknown/unclear path) to specify the pre-crash 


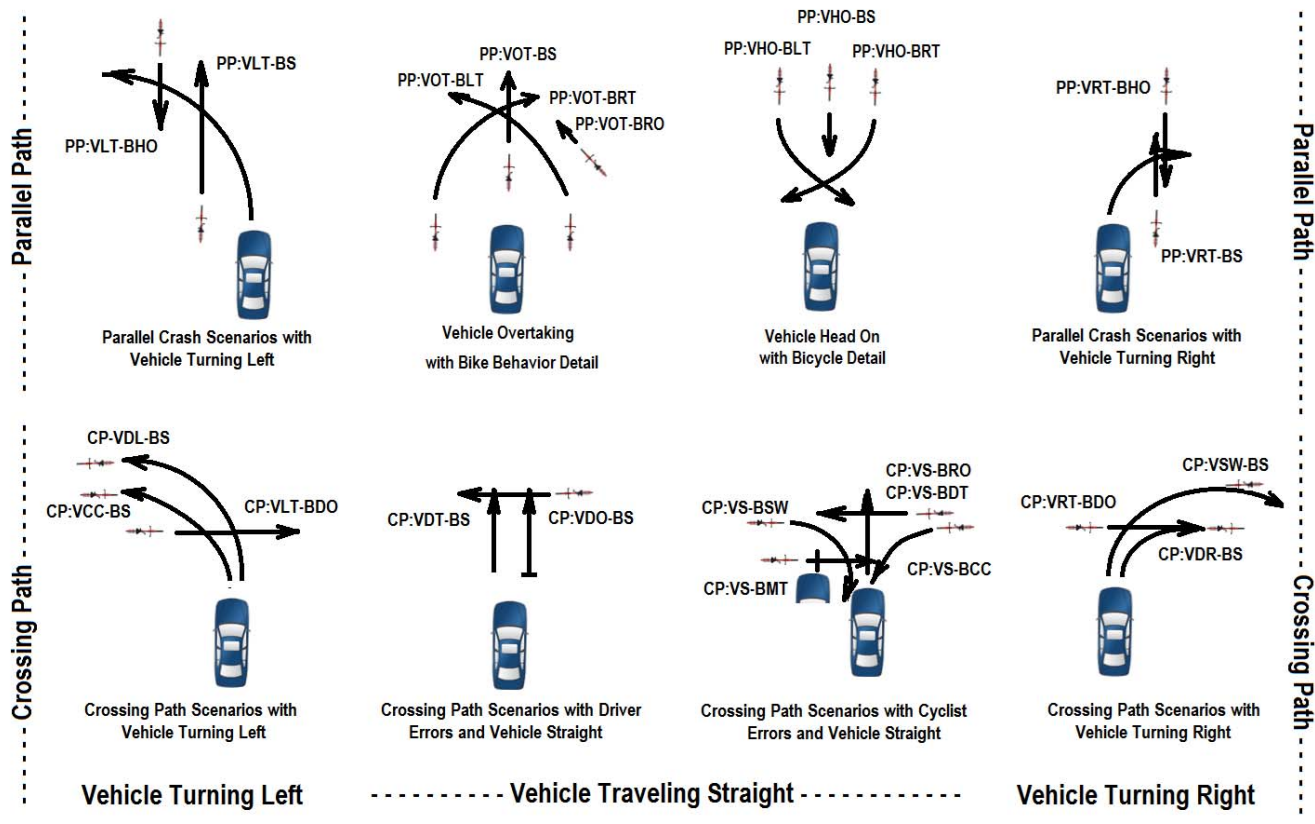

Figure 3 ThreeTier Testable Bicycle Crash Scenarios

paths of the vehicle and bicycle relative to one. UP are those where there is no clear direction to the bicycle for example while a riding in a parking lot. In tier 2 we identify the vehicle motion prior to the crash and use VOT, VHO, VRT, VLT, VS or VDO (to indicate the vehicle is overtaking the bike, approaching the bike head on, turning right or left, or going straight or driving out, stopping then going). Finally, individual elements in the figure incorporate subtleties of bicyclist behaviors and maneuvers. In Figure 3, the three tiers are represented as rows (gross geometry), columns (vehicle action) or individual elements (cyclist behavior).

Our analysis suggests that out of the 24 identifiable bicycle crash scenarios, the most serious scenario, PP:VOT-BS (vehicle striking the bicyclist from behind) involves $26 \%$ of social cost, $34 \%$ of fatalities but only $12 \%$ of the number of crashes. The second most serious crash scenario, CP: VS-BRO (vehicle striking the bicyclist as they ride out in the vehicle path after stopping) involves $17 \%$ of the crashes, $17 \%$ of the fatalities and $17 \%$ of the social cost. This makes sense since vehicles are likely to have slower speeds in areas where they face frequent intersections. Scenarios where the vehicle is turning have much lower potential for harm because vehicle speeds are lower. In addition, CIB systems typically are disabled when the vehicle is under active maneuver such as in a turn. This is clearly stated in the owner's manual as is the relative ineffectiveness in crossing scenarios.

Our analysis focuses on the PP:VOT-BS scenario since it is both the most important, and the vehicle manufacturer claims to have some effectiveness in these situations.

\section{Bicyclist Crash Surrogate}

Our cyclist surrogate is designed to visually look like an adult cyclist, $174 \mathrm{~cm}$ tall (a weighted average of males and females involved in crashes) and appear to weigh (volume) 73 $\mathrm{kg}$ with a BMI of 24 . This is a little chubbier than the Hybrid
III adult male designed in the 1970s as BMI have risen considerably since then with sizing developed from current American anthropometric data in NHANES [7]. The mannequin is covered with a metal fabric skin that provides the same radar cross section at $77 \mathrm{GHz}$ as a human would and rides on a 26" mountain bike [8] and rides on a carrier that is both durable enough to accommodate vehicle impacts and unobtrusive [9]. The developed surrogate is light, durable and unlikely to cause vehicle damage on collision [10]. Instrumentation to predict injuries conflicts with these goals.

\section{An Analysis of Vehicle and Cyclist Speeds}

Unfortunately, bicycle crashes are not systematically investigated and compiled in Federal databases in the US (with methodologies such as the CDS, now CISS). While there are plans to do so, the data are not expected to be available until after 2020. Vehicle speed is clearly a dominant variable in both the extent of cyclist injury and in system effectiveness. It is anticipated that faster vehicle speeds will provide less system reaction time, and lead to greater injuries. The best surrogate for vehicle speed for cyclist crashes is to examine vehicle speed for pedestrian crashes from the most recent systematic study, the Pedestrian Crash Data Study, PCDS [11]. This proxy is intended to identify general characteristics about speed rather than provide exact benefit information.

In previous work we have analyzed several factors that allow better prediction of vehicle traveling speed just prior to pedestrian crashes [12]. Unlike there, there is no source of investigated crashes like PCDS where traveling speed can be reconstructed. Figure 4 shows the speed at impact for those situations in terms of crash frequency (top) and social cost based on injury severity (bottom). This reinforces the notion that speeds for vehicles traveling straight, particularly when they are not at intersections, leading to increased likelihood of serious injury or fatality. 



Figure 4. Vehicle traveling speeds and crash frequency (top) and social costs (bottom) for pedestrian crashes

Our cyclist speed information is generated by examining bicyclists from footage from our naturalistic driving study [10]. For 1000 adult cyclists, median speeds were $12.5 \mathrm{mph}$ with upper and lower quartiles at 16 and $9 \mathrm{mph}$. The mannequin can also reflect leg motion (pedaling) as was also observed during this study and is reflected in mannequin movement.

\section{E. Other Test Factors}

The vast majority of crashes occur during daylight hours. Children are involved primarily in crossing crashes. While a child mannequin was developed, it was not used for this round of testing. Cyclists are relatively unlikely to ride during inclement weather. Obscuring objects are present in only about $9 \%$ of crashes. These are typically widely distributed over most crash types with a common occurrence only where the cyclist is trapped, facing multiple threats (CP: VS-BMT).

\section{System PERformanCE TESTING}

\section{A. Testing and Test Site}

All cyclist crash imminent braking systems were conducted on the $13 \mathrm{~m}$ wide asphalt test track in Columbus, Indiana. 89 tests were performed with usable information under a variety of conditions: varying bicycle speed, vehicle speed, pedaling behavior and cyclist position relative to the vehicle.

\section{B. Econometric Modeling of System Performance}

The performance of a cyclist CIB system must determine a vehicle speed at impact. We use a doubly truncated Tobit model that posits an underlying latent index identifying the challenge posed for detection system and its capacity to meet that challenge. The measure of effectiveness is defined by:

$$
\text { Effectiveness }=\frac{\text { Travel Speed }- \text { Impact Speed }}{\text { Travel Speed }}
$$

This functional form identifies the effects of other variables in the test scenario to be proportional to travel speed. In addition to providing better fits for the data, the model is less subject to problems of heteroskedasticity (the variance of the error term being related to regressors which leads to inefficient estimates and tends to bias standard errors, invalidating confidence intervals and implied hypothesis tests) [13]. We use a maximum likelihood estimator where $y^{*}$ is latent effectiveness,

$$
y *=\beta \cdot x+\varepsilon \quad \text { with } \quad y=\left\{\begin{array}{cc}
1 & \text { if } y *>1 \\
y * & \text { if } 0 \leq y * \leq 1 \\
0 & \text { if } y *<0
\end{array}\right.
$$

$\mathrm{y}$ is observed effectiveness and the log-likelihood function is

$$
\begin{aligned}
& \log \mathscr{L}(\beta, \sigma, \alpha \mid x, y)= \\
& \quad \sum_{y=0} \log \Phi\left(\frac{\alpha+\beta \cdot x}{\sigma}\right)+\sum_{y=1} \log \Phi\left(\frac{1-\alpha-\beta \cdot x}{\sigma}\right)+\sum_{0<y<1} \log \left[\frac{1}{\sigma} \varphi\left(\frac{y-\alpha-\beta \cdot x}{\sigma}\right)\right]
\end{aligned}
$$

Here $\Phi$ and $\varphi$ represent the standard normal cdf and density. A and $\beta$ are regression parameters, and $\sigma$ is the standard deviation of the error. The model estimates for our system effectiveness measure are based on 89 simulated crashes using a vehicle equiped with a cyclist CIB system and our PP:VOT-BS crash scenario. Estimates are in Table 1.

\begin{tabular}{|l|ccc|ccc|}
\hline & \multicolumn{3}{|c|}{ Quadratic Expansion Model } & \multicolumn{3}{c|}{ Linear Expansion Model } \\
variable & estimate & std err & p-val & estimate & std err & p-val \\
\hline intercept & .70563 & .01934 & .002 & .71839 & .30641 & .022 \\
vehicle speed & -.12329 & .00233 & .000 & -.12415 & .22888 & .000 \\
veh speed`2 & -.00387 & .00233 & .101 & -- & -- & -- \\
cyclist speed & .05601 & .02804 & .050 & .17393 & .03492 & .000 \\
cycl speed^2 & -.01374 & .00485 & .004 & -- & -- & -- \\
centered & .52108 & .22265 & .022 & .17993 & .25831 & .530 \\
limb motion & -.00033 & .22126 & .998 & .07474 & .21503 & .729 \\
\hline sigma & .43383 & .06769 & -- & .60314 & .09652 & -- \\
chi-sq (df) & 80.00 & 6 & .000 & 53.72 & 4 & .000 \\
Pseudo-R`2 & .5552 & & & .3728 & & \\
\hline
\end{tabular}

\section{Table 1. Tobit estimates for system performance model}

The objective behind the final models estimated is not to identify individual factors that we can prove are related to system performance but to predict the system performance over a variety of scenarios using the best available predictor. As a result, our analysis maintains some variables in the formulation that are not statistically significant because they provide valuable controls for an unbalanced factorial design and better reflect outcomes in real world implementation. We find that a quadratic model (second order Taylor series in the kernel) fits substantially better than a linear model (increase in Rsquare from .37 to .55 , p-value of .000). Dominant variables in the model are vehicle traveling speed, cyclist speed, and whether the cyclist was centered on the front of the vehicle or offset $25 \%$ of the way from the right front corner (a common point of impact). Limb motion matters little. A graphical representation of performance incorporating vehicle and cyclist speed is presented in Figure 5. Our estimates indicate that the system is most effective for bicycle speeds of approximately 12 $\mathrm{mph}$. In other words, it is well tuned for the kind of riders that were encountered during our naturalistic driving study.

For our scenario, if the vehicle is traveling slower than the bicycle, no crash will occur. We anticipate that the faster the relative speed difference the more serious injuries are likely to be. The difference in vehicle speed and cyclist speed, delta-V, 
has a long history as an injury severity predictor for injuries caused by the inelastic collision with the cyclist $[14,15]$.

Crashes are averted (cyclist delta- $\mathrm{V}$ of 0 ) if the system is effective enough to slow the vehicle to less than the cyclist speed before impact. In Figure 6 . the difference between the solid line and the dashed line shows how much delta- $V$ has been reduced as a result of the CIB system effectiveness.

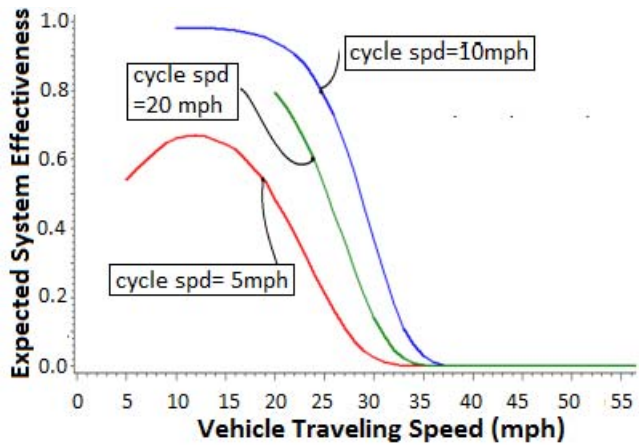

Figure 5. Comparison of system effectiveness for cycling speeds.

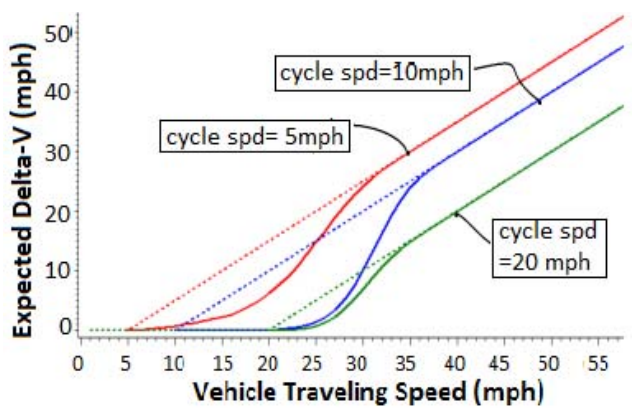

Figure 5. Cyclist Delta-V for vehicle and cyclist traveling speeds

\section{PROXYING BiCYClist CRASH OUtCOMES}

\section{A. Consequence Assessment.}

Several authors have previously estimated the effects of factors on the severity of pedestrian injuries [16,17]. There are relatively few empirical models developed specifically for bicyclists. Among the best work examining both [18], they do not account for key variables such as cyclist speed, crash geometry (e.g., overtaking vs. headon) or, cyclist age (frailty). This makes it difficult to translate this work from the German to the American context. To complement that work we use a detailed model of pedestrian crashes and adjust the results to bicyclists. Our analysis is based on PCDS [18, 19] and includes variables such as non-motorist age, and a geometry regarding how the pedestrian was struck in addition to vehicle speed.

Because of the number of variables, parsimony requires estimating an ordinal probit model predicting the extent of injury as measured by the Maximum Abbreviated Injury Scale (MAIS). Because the investigation of cases in PCDS does not match the distribution of all crashes well (PCDS tended to investigate crashes with more severe consequences more heavily), we weight the observations in our maximum likelihood estimates proportionately to the occurrence of severities in GES for sample (e.g., crash severities that are over-represented get weights of less than 1.0 while crash severities that are underrepresented get weights greater than 1.0). Estimates are in Table 2.

\begin{tabular}{|lrrr|ll|}
\hline \multicolumn{5}{|c|}{ Dependent variable: } \\
\hline Variable & estimate & std err & p-val & & \\
\hline cut point 1 & -0.8244 & .2113 & .000 & & \\
cut point 2 & 2.3088 & .1588 & .000 & & \\
cut point 3 & 3.0021 & .1661 & .000 & Sample Size & \\
cut point 4 & 3.7252 & .1788 & .000 & & \\
cut point 5 & 4.2002 & .1914 & .000 & Log Likelihood & -575.93 \\
cut point 6 & 5.2847 & .2425 & .000 & & \\
vehicle speed & -0.0653 & .0050 & .000 & LR Chi-sq (7 df) & 231.71 \\
ped age & -0.0110 & .0026 & .000 & & \\
with path & -0.3504 & .1373 & .011 & McFadden R^2 & .171 \\
cross paths & -0.2076 & .4923 & .673 & Cox/Snell R`2 & .404 \\
ped speed & -0.0316 & .0405 & .435 & & \\
dark unlit & -0.3224 & .2387 & .177 & & \\
dark lit & -0.0990 & .1180 & .402 & & \\
\hline
\end{tabular}

Table 2. Parameter estimates for ordinal probit model

Variables in the model include vehicle speed, the relative paths (with path has vehicle striking pedestrian from behind), pedestrian speed (standing, walking speed, running); and lighting conditions (daylight is base case).

To simplify exposition, a comparable model is estimated using for pedestrian fatality using the same predictor variables. The parameter estimates are similar to those in Table 2 but without cut-points. The main argument for the usability of these results is that speed at impact for pedestrians is a good proxy for the delta- $\mathrm{V}$ for cyclists. In Figure 6 the probability of fatality is shown for different pedestrians aged 20, 45 and 70. The frailty of a 70 year old compared to a 20 year old is like a $10 \mathrm{mph}$ increase in speed at impact. For a $40 \mathrm{mph}$ delta-V, increases fatality risk from .2 to .5 .

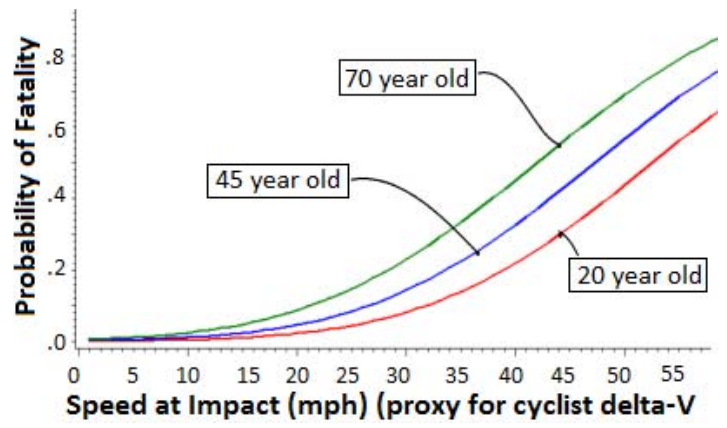

Figure 6. Probability of Fatality as a function of Speed at Impact (a Proxy for Cyclist Delta-V)

\section{B. System integration}

Integrating the model of system performance with the model of injury causation is summarized in Figure 7 for a cyclist traveling speed of $10 \mathrm{mph}$, close to the system's "sweet spot" or most effective cyclist speed. The use of a cyclist CIB system shows benefits for different ages of riders and different vehicle speeds. For a twenty year old cyclist in a crash with a vehicle that is not CIB equipped (solid red line) there is little difference from one with CIB (dashed red line). Similarly, the solid blue and green lines indicate the delta- $\mathrm{V}$ for a 45 year old and 70 year old encountering a vehicle that is not cyclist CIB equipped while the corresponding dashed lines represent the same scenario with a CIB equipped vehicle. This represents a near best case scenario. 


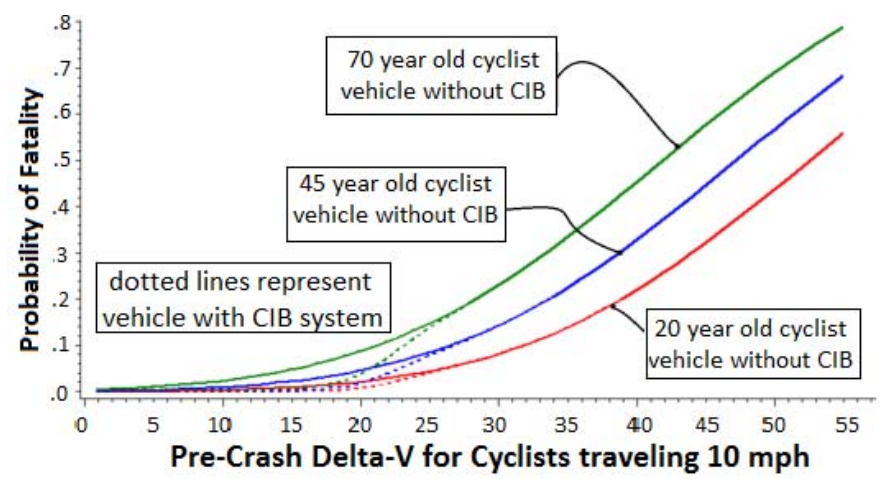

Figure 7. Life-saving potential of cyclist CIB system

The biggest benefits occur when the solid and dashed lines are the farthest apart. This occurs for pre-crash delta-V (closing speeds) of around $18 \mathrm{mph}$ for all age groups. By the time closing speeds are $25 \mathrm{mph}$ the system loses any effectiveness (the lines converge). These cases occur when vehicle speed is around $28 \mathrm{mph}$ for maximum benefit and by $35 \mathrm{mph}$ the life saving benefits are exhausted for all age groups.

For younger bicyclists, in the delta-V range where the PCS system is effective, the probability of fatality is small enough, (only two or three percent), that relatively few lives will be saved by such a system. For older riders, the probability of fatality is nearly three times as high in delta- $\mathrm{V}$ ranges where the system is effective, with much more lifesaving. For a cyclist traveling at $10 \mathrm{mph}$, the maximum benefit is obtained with a closing speed of $19 \mathrm{mph}$. The reduction in fatalities at this most effective speed is $1.9 \%$ reduction in the probability of a 20 year old fatality, $2.7 \%$ reduction for a 45 year old, and $5.2 \%$ for a 70 year old. As older Americans continue cycling as a way to keep active and fit, this will be increasingly important.

\section{SUMmary AND CONCLUSIONS}

In this paper we identified the most important bicyclist crash scenarios for crash imminent braking systems. Our tested scenario involved a crash geometry that is responsible for approximately a quarter of cyclist social cost and a third of cyclist fatalities in single light vehicle crashes. Its primary contribution is that it is based on actual test track data, rather than optimistic simulated performance. As such it embodies key crash features such as time to collision, vehicle speed, and importantly, the speed that cyclists bring to the collision.

We developed a model of cyclist injuries based on injuries incurred by pedestrians, and adapted the results using delta- $\mathrm{V}$ in pace of vehicle speed at impact. We intend this as suggestive rather than definitive since it allows us to incorporate other modifying variables such as age and crash geometry.

The generality of our results is limited in that they examine only one scenario (albeit the most deadly) and only one manufacturer's vehicle. Still, they are suggestive about the limited co-benefits available in bicyclist CIB systems compared to pedestrian systems. Further, the paper lays out a methodology for the evaluation of other systems and scenarios given the limited bicyclist data available. The design and enhancement of CIB systems, like our analysis, remains a work in progress.

\section{ACKNOWLEDGMENT}

The authors thank Toyota's Collaborative Safety Research Center for support to the Transportation Active Safety Institute at Indiana University-Purdue University Indianapolis and the Indiana University Transportation Research Center. Additional support provided by the Crash Imminent Safety University Transportation Center at Ohio State University.

\section{REFERENCES}

[1] Good, D. H., S Chien, L Li, K Krutilla and Y Chen (2015) Preliminary Benefit Analysis for Pedestrian Crash Imminent Braking Systems. IEEE 18th International Conference on Intelligent Transportation Systems 1123-8.

[2, 3, 4] USDOT, NHTSA (2012-17) Traffic Safety Facts: Bicyclists and Other Cyclists 2010-15. Publication numbers DOT HS 811 624, DOT HS 812151 and DOT HS 812151

[5] World Health Organization (2015) Global Status Report on Road Safety United Nations, 2015.

[6] USDOT (2014) Memorandum from Under Secretary RE Treatment of the Economic Value of a Statistical Life in Departmental Analyses - 2014 Interim Adjustment

[7] Centers for Disease Control and Prevention (2005-2011). National Health and Nutrition Examination Survey Data. Hyattsville, MD: U.S. Department of Health and Human Services,

[8] Belgiovane, D.J and C-C Chen (2016) RCS Measurements of Bicycles and Human Riders for Automotive Radar No 2016-01-0168 SAE Technical Paper.

[9] Rini Sherony, Qiang Yi, Stanley Chien, Jason Brink, Mohammad Almutairi, Keyu Ruan, Wensen Niu, Lingxi Li, Yaobin Chen, and Hiroyuki Takahashi, "Development of a Bicycle Carrier for Bicyclist Pre-Collision System Evaluation,” No. 2016-01-1446, SAE Technical Paper, 2016.

[10] Chen, Y., X. Chien, L. Li, H. Yokota, L. Christopher, J. Zheng, R. Tian, D. Good, C-C. Chen. (2016) Development and Testing of Standard Bicyclist Crash Scenarios. Final Report. Transportation Active Safety Institute. 219pp.

[11] NASS Pedestrian Crash Data Study 1994-1998, version 03-Mar-2001, The University of Michigan Transportation Research Institute

[12] Good, D and Abrahams, R. (2014) Vehicle Speeds for Pedestrian PreCrash System Test Scenarios. ITSC 2014 Proceedings.

[13] Madalla, G. (1983) Limited Dependent Variables in Econometrics. Cambridge University Press

[14] Shelby, S. 2011. Delta-V as a Measure of Traffic Conflict Severity, Transportation Research Board annual Meeting, 2011.

[15] Svensson, A. and Hyden, C., Estimating the severity of safety related behaviour. Accident Analysis and Prevention, Vol. 38, pp. 379-385, 2006.

[16] Rosen, E., \& Sander, U. (2009). Pedestrian fatality risk as a function of car impact speed. Accident Analysis \& Prevention, 41(3), 536-542.

[17] Wisch M, Seiniger P, Pastor C, Edwards M, Visvikis C. (2013) Scenarios and weighting factors for pre-crash assessment of integrated pedestrian safety systems. 2013

[18] Rosén, E. (2013) Autonomous Emergency Braking for Vulnerable Road Users, IRCOBI Conference 2013 (paper IRC-13-71)

[19] Simms, C. and D. Wood (2009) Pedestrian and Cyclist Imact: A Biomechanical Perspective (Springer). 